genotoxic potential. Even in its hydrolysed form it is not hazardous to the effluent water.

\section{Summary}

Comprehensive information on toxicological and ecological properties is available for the majority of commercial colorants. With increasing information it became evident that many of the colorants do not pose a pronounced toxicological hazard. It is now possible to focus further attention to those groups of compounds which possess a specific toxicological behavior. Beside adequate labelling and exposure minimization, attempts are made to understand and study their modes of action in more detail.

General considerations on colorants:

- Evaluation in the event of azo dyes possibly being reduced to mutagenic or cancerogenic aromatic amines.

- Azo dyes which could form amino sulphonic acids as hypothetic metabolites are not carcinogenic.
- Reactive dyes and disperse dyes should be evaluated with regard to a possible sensitizing potential.

- Azo pigments based on 3,3'-dichlorobenzidine have proved to be not toxic after acute or repeated exposure and proved to be not mutagenic and not cancerogenic due to their non-bioavailability.

- Organic pigments in general, and in particular when extremely insoluble due to their chemical structure are of very little toxicological and ecological relevance.

- To a certain extent auxiliaries must be made responsible for toxic effects in the commercial colorants.

- Hazard assessment of the mutagenicity with aromatic nitro and amino compounds (colorants) cannot rely on Ames test data only.

Once the toxicological and ecological profile has been established (hazard assessment) reliable risk assessment must include the exposure situation. Control and minimization of non-intended exposure and knowledge of the chemical characteristics will guarantee a good hygiene standard and safe handling of dyes and pigments at the work place (risk management).

Improvement in recycling processes and attempts to decrease waste burden, in combination with knowledge of ecological behavior, will further reduce adverse effects on the environment.

[I] R. Anliker, I. Soc. Dyers Col. 1979, 95, 317.

[2] K.H. Leist, Ecotox. Environm. Safety 1982, 6 , $457-463$.

[3] ETAD Report T 2028, 1990.

[4] R. Anliker, E.A. Clarke, Chemosphere 1980, 9 , 595-609.

[5] R. Anliker, Textilveredlung 1983, /8, 92-98.

[6] E. Longstaff, Dyes Pigments 1983, 4, 243-304.

[7] R. Anliker, in 'Toxic Hazard Assessment of Chemicals', Ed. M. Richardson, Royal Soc. of Chemistry, London, 1986, p. 166-187.

[8] D. L. v. Goethem et al., ETAD internal report, 1990.

[9] R. Jung, D. Steinle, R. Anliker, ETAD Report T 2021, in press (Food and Chemical Toxicology), 1991.

[10] Hoechst $A G$, unpublished.

[11] ETAD Report T 2017, unpublished

[12] J. Le, R. Jung, N. Kramer, Food Chem. Toxicol. $1985,23,695-700$.

\title{
Methämoglobin-Bildung und Hämoglobin-Bindung bei aromatischen Aminen
}

\section{Hans-Günter Neumann*}

Die Geschichte der synthetischen Farbmittel ist wesentlich mit der Chemie des Anilins, des Nitrobenzols und der davon abgeleiteten Arylamine verknüpft. Der Apotheker Unverdorben stellte 1826 Anilin erstmals durch Kalkdestillation aus natürlichem Indigo dar. Synthetisch wird es durch Reduktion von Nitrobenzol auf einem Weg gewonnen, auf dem auch Hydrazobenzol und aus diesem durch Umlagerung Benzidin erhalten wird. Diazotieren eröffnet den Weg zu den Azofarbmitteln. Damit ist eine Entwicklung skizziert, die immer neue Anwendungen erschloss und so stürmisch verlief, dass die damit verbundenen Gefahren für die Gesundheit bei Herstellung und Anwendung nur wenig beachtet wurden. Die Geschichte der synthetischen Farbmittel is damit auch eine Geschichte der Verdrängungen und sie zeigt, wie die Beschäftigung mit den Gesundheitsgefahren und den dar-

*Korrespondenz: Prof. Dr. H.-G. Neumann Institut für Pharmakologie und Toxikologie der Universität Würzburg

Versbacherstr. 9

D-8700 Würzburg aus zu ziehenden Schlussfolgerungen bisher immer hinter der Entwicklung zurückgeblieben ist.

\section{Die Erzeugung von Methämoglobin}

Bereits 1863 wurde die akut toxische Wirkung von Anilin und Nitrobenzol festgestellt [1]: Beide erzeugen Methämoglobin, jene Form des Hämoglobins, in der aufgrund der Oxidation des zweiwertigen zum dreiwertigen Eisen in der Hämgruppe die Fähigkeit zur Bindung molekularen $\mathrm{O}_{2}$ verlorengegangen ist. Auch die Farbe des roten Blutfarbstoffs verändert sich dabei, und erste Vergiftungserscheinungen sind besonders an Lippen und unter den Fingernägeln erkennbar. Daher stammt der Begriff der Blausucht (Zyanose). Die sogenannten «Blue boys» waren in der Anilin-Fabrikation keine Seltenheit und eine Art von Gesundheitsschutz bestand lediglich darin, besonders gesunde Arbeiter für diese Tätigkeit auszusuchen.

Nach 1886 wurde wiederholt über die
Vergiftung von Säuglingen berichtet, deren Windeln mit. Anilin-haltiger Wäschetinte markiert waren [2]. Dies zeigt einerseits die gute Aufnahme uber die Haut, andererseits die besondere Empfindlichkeit in diesem Alter, in dem die Rückreduktion des Methämoglobins zu Hämoglobin, eine physiologische Schutzreaktion, noch nicht voll ausgebildet ist.

Bereits 1891 wurde 4-Aminobiphenyl als stark Methämoglobin-erzeugend beschrieben [3], und bald danach diese akut toxische Wirkung als allgemeine Eigenschaft der Substanzklasse erkannt. Weitere Marksteine sind die Aufdeckung dieser Eigenschaft 1935 für Prontosil (Chrysoidin sulfonamid) [4], das ebenso wie das zur Schönung von Pflanzenfetten verwendete Buttergelb ( $N, N$-Dimethylaminoazobenzol) [5] reduktiv zu Arylamin-Komponenten gespalten wird. Für die antibakterielle Wirkung von Prontosil ist das freigesetzte Sulfanilamid, die Muttersubstanz der Sulfonamide, verantwortlich. Bei seiner therapeutischen Anwendung tritt Methämoglobinämie nicht selten als Nebenwirkung auf. Durch den Verzehr Buttergelb-gefärbter Margarine wurden zwar beim Menschen keine Cyanosen ausgelöst, aber im Tierversuch konnte die Methämoglobin-Bildung durch zahlreiche Azo-Verbindungen gezeigt werden [6]. Die vorangehende Azo-Spaltung wurde zunächst als NADPH-abhängige Reaktion mit Rattenleberhomogenaten nachgewiesen [7]. Erst 1962 wurde erkannt, dass bestimmte Azofarbmittel (FD and CRed No. 2 und 4; FD and C Yellow No 6) auch durch Darmbakterien reduktiv zu den Kupplungskomponenten gespalten werden [8]. In vivo spielt 
genotoxic potential. Even in its hydrolysed form it is not hazardous to the effluent water.

\section{Summary}

Comprehensive information on toxicological and ecological properties is available for the majority of commercial colorants. With increasing information it became evident that many of the colorants do not pose a pronounced toxicological hazard. It is now possible to focus further attention to those groups of compounds which possess a specific toxicological behavior. Beside adequate labelling and exposure minimization, attempts are made to understand and study their modes of action in more detail.

General considerations on colorants:

- Evaluation in the event of azo dyes possibly being reduced to mutagenic or cancerogenic aromatic amines.

- Azo dyes which could form amino sulphonic acids as hypothetic metabolites are not carcinogenic.
- Reactive dyes and disperse dyes should be evaluated with regard to a possible sensitizing potential.

- Azo pigments based on 3,3'-dichlorobenzidine have proved to be not toxic after acute or repeated exposure and proved to be not mutagenic and not cancerogenic due to their non-bioavailability.

- Organic pigments in general, and in particular when extremely insoluble due to their chemical structure are of very little toxicological and ecological relevance.

- To a certain extent auxiliaries must be made responsible for toxic effects in the commercial colorants.

- Hazard assessment of the mutagenicity with aromatic nitro and amino compounds (colorants) cannot rely on Ames test data only.

Once the toxicological and ecological profile has been established (hazard assessment) reliable risk assessment must include the exposure situation. Control and minimization of non-intended exposure and knowledge of the chemical characteristics will guarantee a good hygiene standard and safe handling of dyes and pigments at the work place (risk management).

Improvement in recycling processes and attempts to decrease waste burden, in combination with knowledge of ecological behavior, will further reduce adverse effects on the environment.

[I] R. Anliker, I. Soc. Dyers Col. 1979, 95, 317.

[2] K.H. Leist, Ecotox. Environm. Safety 1982, 6 , $457-463$.

[3] ETAD Report T 2028, 1990.

[4] R. Anliker, E.A. Clarke, Chemosphere 1980, 9 , 595-609.

[5] R. Anliker, Textilveredlung 1983, /8, 92-98.

[6] E. Longstaff, Dyes Pigments 1983, 4, 243-304.

[7] R. Anliker, in 'Toxic Hazard Assessment of Chemicals', Ed. M. Richardson, Royal Soc. of Chemistry, London, 1986, p. 166-187.

[8] D. L. v. Goethem et al., ETAD internal report, 1990.

[9] R. Jung, D. Steinle, R. Anliker, ETAD Report T 2021, in press (Food and Chemical Toxicology), 1991.

[10] Hoechst $A G$, unpublished.

[11] ETAD Report T 2017, unpublished

[12] J. Le, R. Jung, N. Kramer, Food Chem. Toxicol. $1985,23,695-700$.

\section{Methämoglobin-Bildung und Hämoglobin-Bindung bei aromatischen Aminen}

\section{Hans-Günter Neumann*}

Die Geschichte der synthetischen Farbmittel ist wesentlich mit der Chemie des Anilins, des Nitrobenzols und der davon abgeleiteten Arylamine verknüpft. Der Apotheker Unverdorben stellte 1826 Anilin erstmals durch Kalkdestillation aus natürlichem Indigo dar. Synthetisch wird es durch Reduktion von Nitrobenzol auf einem Weg gewonnen, auf dem auch Hydrazobenzol und aus diesem durch Umlagerung Benzidin erhalten wird. Diazotieren eröffnet den Weg zu den Azofarbmitteln. Damit ist eine Entwicklung skizziert, die immer neue Anwendungen erschloss und so stürmisch verlief, dass die damit verbundenen Gefahren für die Gesundheit bei Herstellung und Anwendung nur wenig beachtet wurden. Die Geschichte der synthetischen Farbmittel is damit auch eine Geschichte der Verdrängungen und sie zeigt, wie die Beschäftigung mit den Gesundheitsgefahren und den dar-

*Korrespondenz: Prof. Dr. H.-G. Neumann Institut für Pharmakologie und Toxikologie der Universität Würzburg

Versbacherstr. 9

D-8700 Würzburg aus zu ziehenden Schlussfolgerungen bisher immer hinter der Entwicklung zurückgeblieben ist.

\section{Die Erzeugung von Methämoglobin}

Bereits 1863 wurde die akut toxische Wirkung von Anilin und Nitrobenzol festgestellt [1]: Beide erzeugen Methämoglobin, jene Form des Hämoglobins, in der aufgrund der Oxidation des zweiwertigen zum dreiwertigen Eisen in der Hämgruppe die Fähigkeit zur Bindung molekularen $\mathrm{O}_{2}$ verlorengegangen ist. Auch die Farbe des roten Blutfarbstoffs verändert sich dabei, und erste Vergiftungserscheinungen sind besonders an Lippen und unter den Fingernägeln erkennbar. Daher stammt der Begriff der Blausucht (Zyanose). Die sogenannten «Blue boys» waren in der Anilin-Fabrikation keine Seltenheit und eine Art von Gesundheitsschutz bestand lediglich darin, besonders gesunde Arbeiter für diese Tätigkeit auszusuchen.

Nach 1886 wurde wiederholt über die
Vergiftung von Säuglingen berichtet, deren Windeln mit. Anilin-haltiger Wäschetinte markiert waren [2]. Dies zeigt einerseits die gute Aufnahme uber die Haut, andererseits die besondere Empfindlichkeit in diesem Alter, in dem die Rückreduktion des Methämoglobins zu Hämoglobin, eine physiologische Schutzreaktion, noch nicht voll ausgebildet ist.

Bereits 1891 wurde 4-Aminobiphenyl als stark Methämoglobin-erzeugend beschrieben [3], und bald danach diese akut toxische Wirkung als allgemeine Eigenschaft der Substanzklasse erkannt. Weitere Marksteine sind die Aufdeckung dieser Eigenschaft 1935 für Prontosil (Chrysoidin sulfonamid) [4], das ebenso wie das zur Schönung von Pflanzenfetten verwendete Buttergelb ( $N, N$-Dimethylaminoazobenzol) [5] reduktiv zu Arylamin-Komponenten gespalten wird. Für die antibakterielle Wirkung von Prontosil ist das freigesetzte Sulfanilamid, die Muttersubstanz der Sulfonamide, verantwortlich. Bei seiner therapeutischen Anwendung tritt Methämoglobinämie nicht selten als Nebenwirkung auf. Durch den Verzehr Buttergelb-gefärbter Margarine wurden zwar beim Menschen keine Cyanosen ausgelöst, aber im Tierversuch konnte die Methämoglobin-Bildung durch zahlreiche Azo-Verbindungen gezeigt werden [6]. Die vorangehende Azo-Spaltung wurde zunächst als NADPH-abhängige Reaktion mit Rattenleberhomogenaten nachgewiesen [7]. Erst 1962 wurde erkannt, dass bestimmte Azofarbmittel (FD and CRed No. 2 und 4; FD and C Yellow No 6) auch durch Darmbakterien reduktiv zu den Kupplungskomponenten gespalten werden [8]. In vivo spielt 
die Spaltung von Azo-Verbindungen im Darm die wichtigste Rolle.

1950 führte die Beschäftigung mit dem Wirkungsmechanismus der Methämoglobinbildung zu der Entdeckung, dass Phenylhydroxylamin besonders stark wirksam ist. Die Oxidation von Anilin zu Phenylhydroxylamin und Nitrosobenzol im Stoffwechsel wurde 1959 durch Kiese beim Hund nachgewiesen [9]. Damit war der Schlüssel zum Verständnis der Methämoglobin-Bildung gefunden. Heute wissen wir, dass die sowohl aus Arylaminen als auch aus Nitroaromaten im Stoffwechsel gebildeten $\mathrm{N}-\mathrm{Hy}$ droxy-arylamine im Erythrozyten durch Oxyhämoglobin zu Nitroso-Derivaten oxidiert werden. In einer Kooxidationsreaktion wird dabei gleichzeitig das $\mathrm{Fe}^{2+}$ des Hämoglobins zum $\mathrm{Fe}^{3+}$ des Methämoglobins oxidiert. Beide Oxidationsprodukte können in vivo wieder reduziert werden, sodass ein Kreisprozess in Gang gesetzt wird, bei dem ein Molekül des Hydroxylamins zahlreiche Hämoglobin-Moleküle oxidiert. Diese beiden Reaktionen verlaufen jedoch unterschiedlich schnell, sodass verhältnismässig geringe Mengen des Methämoglobinbildners einen starken Effekt auslösen können.

\section{Das Konzept der metabolischen Akti- vierung}

Kurz nach der Entdeckung der Oxidation von Anilin wurde im Arbeitskreis des Ehepaars Miller die $\mathrm{N}$-Hydroxylierung von 2-Acetylaminofluoren im Stoffwechsel der Ratte nachgewiesen und als metabolische Aktivierungsreaktion gedeutet [10]. Damit war die N-Oxidation von Arylaminen zum Schnittpunkt der Entwicklung bei der Aufklärung der Mechanismen sowohl der Methämoglobinbildung als auch der Krebsentstehung durch Arylamine geworden. Das Konzept der metabolischen Aktivierung war formuliert und hat die weitere Forschung grundlegend bestimmt: Im Stoffwechsel werden Fremdstoffe zwar überwiegend inaktiviert und ausscheidbar gemacht, es entstehen dabei aber auch reaktionsfähige Zwischenstufen, die mit Zellbestandteilen reagieren und sowohl reversible als auch irreversible Veränderungen erzeugen können. Für Arylamine und Nitroaromaten stehen dabei die N-Hydroxy-Derivate im Mittelpunkt. Sie können durch Konjugation in einem zweiten Schritt weiter aktiviert werden. Dadurch wird die Bildung der Nitrenium-Ionen erleichtert, die für die mutagene Wirkung dieser Substanzen verantwortlich sind.

\section{Die Farbstoffe in der chemischen Kanzerogenese}

Die Geschichte der Erforschung der chemischen Kanzerogenese ist eng mit den Farbstoffen verknüpft. Rehn beobachtete 1895 bei Fuchsinarbeitern das gehäufte
Auftreten von Blasentumoren [11]. Drei von 45 Arbeitern einer Anilin-Fabrik entwickelten diese Geschwülste. Zur Herstellung des Triphenylmethanfarbstoffs werden Anilin, o-Toluidin und p-Toluidin mit Nitrobenzol in Gegenwart von Salzsäure oxidiert. Die für die Arylamine charakteristischen Blasentumoren sind deshalb unter dem Begriff «Anilinkrebs» in die Literatur eingegangen, obwohl Anilin selbst, wenn überhaupt, nur einen geringen Anteil an der Wirkung hat. Bis heute werden Blasentumoren bei Arylamin-exponierten Arbeitern beobachtet.

Rehn's Befund war in mehrfacher Hinsicht bedeutsam. Zum ersten Mal wurde ein systemisch, d.h. nicht am Ort der unmittelbaren Stoffeinwirkung entstandener Tumor mit einer äusseren Exposition in Zusammenhang gebracht. Arylamine wurden damit zur ersten chemisch definierte Substanzklasse unter den chemischen Kanzerogenen. Ausserdem plädierte Rehn bereits zu diesem Zeitpunkt für eine geplante Krebsprävention.

Die erste chemisch definierte Substanz, die in die Krebsforschung eingeführt wurde, war 1906 das Scharlachrot [12]. Mit der reinen Substanz konnten schliesslich nach oraler Verabfolgung Lebertumoren bei der Ratte erzeugt werden [13]. Mit Buttergelb wurden durch Druckrey und Küpfmüller [14] die ersten Dosis-Wirkungs-Beziehungen bei Kanzerogenen aufgestellt. Sie führten zur Formulierung der $c \times t=$ konst.-Beziehung (Dosis $\times$ Latenzeit $=$ konst.).

Die Entwicklung der Azo-Farbmittel hatte durch die Verwendung von Benzidin als Kupplungskomponente einen enormen Aufschwung erlebt. Schon frühzeitig war aber auch das Auftreten von Harnblasentumoren bei Arbeitern in der Farbmittelindustrie auf Benzidin-Exposition zurückgeführt worden [15]. Experimentell wurde die kanzerogene Wirkung von Benzidin 1950 nachgewiesen [16]. Vorschriften zum Schutz der Arbeiter wurden z.B. in den USA aber erst 1972-1974 erlassen. Die Herstellung der Amine sollte kontrolliert, die Exposition durch Einführung geschlossener Systeme vermieden werden [17].

Etwa die Hälfte der 3000 im Handel befindlichen Farbmittel sind Azo-Verbindungen, 433 enthalten Benzidin oder Benzidinkongenere als Kupplungskomponente [18]. Daneben werden zahlreiche andere Arylamine in der Synthese verwendet. Die Frage nach der kanzerogenen Wirkung der fertigen Azo-Farbmittel wurde 1975 durch eine Beobachtung von Rinde und Troll aufgeworfen [19]. Diese Autoren wiesen die Freisetzung von Benzidin und Monoacetylbenzidin im Stoffwechsel von vier Benzidin-abgeleiteten Azo-Farbstoffen bei Affen nach. Bei dem Test auf subchronische Toxizität zur Vorbereitung eines Kanzerogeneseversuchs mit Ratten wurden mit Direct Blue 6, Direct Black 38 und Direct Brown 95 schon nach fünf Wochen erste Tumoren beobachtet. So eine kurze Latenzzeit war bis dahin überhaupt noch nicht bekannt geworden. Nachdem Benzidin im Harn nachge- wiesen worden war, wurde eine ZweijahresStudie zur Prüfung auf krebserzeugende Wirkung überhaupt nicht mehr angesetzt [20]. Im Harn von Hunden wurde nach Verabfolgung sechs weiterer Azo-Farbstoffe Benzidin in namhaften Mengen gefunden. Auch im Harn von Arbeitern, die mit verschiedenen Azo-Farbmitteln Kontakt hatten, liess sich Benzidin nachweisen [21]. Aus diesen Beobachtungen ergab sich der inzwischen erhärtete Verdacht, dass grundsätzlich mit der Freisetzung der Kupplungskomponenten im Stoffwechsel durch reduktive Spaltung der Azo-Gruppe gerechnet werden muss. Dabei kann es durchaus quantitative, u.a. auch Spezies-bedingte Unterschiede geben, aber in einigen Fällen ist die vollständige Spaltung nachgewiesen worden. Eine Ausnahme stellen bisher die praktisch unlöslichen Pigmente dar.

Nach einer Dokumentation der ETAD [22] sind bisher 148 Azo-Farbmittel auf kanzerogene Wirkung getestet worden. Davon waren 34 Tests postiv, 95 negativ und 19 nach Meinung der Autoren nicht eindeutig. Dabei entsprach die Mehrzahl der Untersuchungen nicht den heute üblichen Anforderungen. Es finden sich jedoch in allen Gruppen der nach chemischen und pharmakologischen Gesichtspunkten unterteilten AzoFarbmittel, mit Ausnahme der Pigmente, Vertreter, die kanzerogen sind oder bei denen aus «inadäquaten Versuchen» Hinweise auf eine solche Wirkung abgeleitet werden können. Es liegt deshalb nahe, die Farbmittel im Hinblick auf die Freisetzung kritischer Arylamine zu beurteilen.

Nach einem 1990 in die MAK-Werte Liste der Bundesrepublik Deutschland aufgenommenen Abschnitt sollen demzufolge alle Azo-Farbmittel so gehandhabt werden, «als ob sie eingestuft wären, wie es der kanzerogenen bzw. kanzerogen verdächtigen Aminkomponente entspricht». Wenn also als Kupplungskomponente Benzidin im Molekül enthalten ist, soll dieses Farbmittel wie ein A1-Stoff (erwiesenermassen für den Menschen kanzerogen) gehandhabt werden [23].

Vielfach wird diese Lösung deshalb als unbefriedigend angesehen, weil im Tierversuch getestete und nicht-kanzerogen gefundene Azo-Farbmittel miterfasst werden. Ein negatives Testergebnis beweist in solchen Fällen jedoch nicht, dass das Amin nicht freigesetzt wird, sondern lediglich, dass die dadurch erzielte Dosis unterhalb des «no observable effect levels» gelegen hat. Da bei gentoxischen Arylaminen ein «no effect level» nicht bestimmt werden kann, bleibt das Minimierungsgebot in Kraft, d.h. die Exposition muss so niedrig wie möglich gehalten werden (ALARA-Prinzip = as low as reasonably achievable).

\section{Expositionskontrolle durch Häınoglo- bin-Addukte}

Im Zusammenhang mit Azo-Farbmitteln wird damit die Frage aufgeworfen, wie 
die innere Exposition durch ein erst nach der Aufnahme freigesetzes gesundheitsgefährdendes Arylamin und die im Stoffwechsel daraus entstehenden Aktivierungsprodukte nachgewiesen und quantitativ bestimmt werden können (Expositionskontrolle), und wie daraus auf ein mit dieser Exposition verbundenes Risiko geschlossen werden kann (Risikobeurteilung).

Der Nachweis der Arylamine im Harn ist sehr unempfindlich, weil nur ein kleiner Bruchteil des unmetabolisierten Amins im Harn erscheint und die Konzentration nach der Exposition rasch abnimmt. Auch die Erhöhung der Methämoglobin-Spiegel im Blut ist zum indirekten Nachweis des Auftretens von $\mathrm{N}$-Hydroxy-arylaminen herangezogen worden. Wegen der relativ raschen Reduktion zum Hämoglobin hängt die Bestimmung aber sehr stark vom Zeitpunkt der Probenahme ab. Demgegenüber erweist sich die Bestimmung eines Reaktionsproduktes, das bei der Umsetzung des im Zuge der Methämoglobin-Bildung entstehenden Nitroso-Derivats mit der SH-Gruppe eines $\mathrm{Cy}$ stein-Restes im Hämoglobin als vorteilhaft. Nach innermolekularer Umlagerung entsteht dabei ein Sulfinsäureamid, das in vivo stabil ist und nach Isolierung des Hämoglobins aus einer Blutprobe hydrolytisch gespalten werden kann. Dabei wird das Ausgangsamin freigesetzt. Es kann extrahiert und quantitativ bestimmt werden [24]. Da die Erythrozyten beim Menschen eine Lebensdauer von 120 Tagen haben und die Elimination des Adduktes wahrscheinlich weitgehend an den Abbau der Erythrozyten gebunden ist, stellt das Hämoglobin-Addukt ein über einen weiten Zeitraum integrierendes Dosimeter dar.

Anilin und Nitrobenzol liefern das gleiche Addukt, die Exposition kann also über die gleiche Analytik kontrolliert werden. In beiden Fällen ist für die akute Toxizität auch der gleiche Metabolit, das Phenylhydroxylamin, verantwortlich. Folgerichtig wurde für beide Substanzen ein gleicher Biologischer-Arbeitsstoff-Toleranzwert (BAT-Wert) auf der Basis des aus dem Hämoglobin-Addukt freisetzbaren Anilins ab-

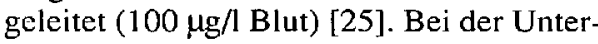
suchung von Arbeitern, die unter MAKBedingungen und vergleichbar Anilin-exponiert waren, liessen sich die gefundenen Werte zwanglos zwei Gruppen zuordnen: den durch einen Enzympolymorphismus unterschiedenen «langsamen und schnellen Acetylierern» [26]. Bei geringer Exposition waren die Hämoglobin-Addukte nur bei den langsamen Acetylierern, also der Gruppe, die auch bei der Methämoglobin-Bildung empfindlicher reagiert, signifikant erhöht. Das unterstreicht die Bedeutung dieses $\mathrm{Pa}$ rameters als eines die individuelle Handhabung einer äusseren Dosis berücksichtigenden Dosimeters. Der oben definierte BATWert schützt den Empfindlichen besser als die Kontrolle der äusseren Exposition oder die Bestimmung der tatsächlich aufgenommenen Dosis durch die Analyse der Ausgangsverbindung in Blut oder Harn.
Der auf die äussere Dosis bezogene Anteil eines Arylamins oder Nitroaromaten, der an Hämoglobin bindet, kann als $\mathrm{Hb}$ Bindungsindex (HBI) ausgedrückt werden. Für eine Reihe von monozyklischen Arylaminen unterscheidet sich der HBI im Tierversuch an der Ratte mit einem Faktor 1000 ganz erheblich [27]. Die Bioverfügbarkeit des für die akute Toxizität und bei kanzerogenen Verbindungen auch für die gentoxische Wirkung verantwortlichen primären Aktivierungsproduktes ist also sehr unterschiedlich. Da dies hauptsächlich pharmakokinetische Gründe hat, sind für ein bestimmtes Amin nicht nur die bereits angesprochenen individuellen Unterschiede sondern vor allem auch solche zwischen verschiedenen Spezies zu erwarten.

Im Zusammenhang mit den Farbmitteln interessiert vor allem die Hämoglobin-Bindung von Benzidin und Benzidin-Abkömmlingen. Bei der Ratte nimmt der HBI von Benzidin (24.3), über 3,3'-Dichlorbenzidin (3.5), 3,3'-Dimethoxybenzidin (2.7) zum 3,3'-Dimethylbenzidin (1.9 $\mathrm{mmol} / \mathrm{mol} \mathrm{Hb} /$ dose $(\mathrm{mmol} / \mathrm{kg}))$ deutlich ab [28]. Bei diesen bifunktionellen Aminen wird als Spaltprodukt des Hämoglobin-Adduktes ausser dem Diamin auch die monoacetylierte Verbindung erhalten. Das Verhältnis ist substanzspezifisch und dosisabhängig. Das unterstreicht die Rolle der Acetylierung im Stoffwechsel dieser Substanzklasse. Im Falle des Benzidins wird als Spaltprodukt auch 4-Aminobiphenyl gefunden, ein Hinweis auf eine neuartige Stoffwechselreaktion, bei der eine aromatisch gebundene Aminogruppe entfernt wird. Mit 3,3',5,5'-Tetramethylbenzidin kann kein Addukt nachgewiesen werden [28]. Das belegt, dass diese Verbindung entweder im Stoffwechsel deutlich schlechter $\mathrm{N}$-oxidiert wird und/oder, dass ein entsprechendes Hydroxylamin schlechter weiterreagiert. Das erklärt auch die Unwirksamkeit im Test auf mutagene Wirkung. Diese Befunde unterstützen die Verwendung dieses Benzidin-Derivates als weniger gefährlichen Ersatz für andere Benzidin-Derivate beim Nachweis von Blutspuren in einem üblichen Farbtest.

Nachdem die Hämoglobin-Bindung von Benzidin und einigen seiner Derivate gezeigt ist, eröffnet sich eine Möglichkeit die Bioverfügbarkeit der entsprechenden Spaltprodukte aus Azo-Farbmitteln in vivo zu bestimmen. So lassen sich nach oraler Gabe von Direkt Rot 28 bei der Ratte die entsprechenden Benzidin-Addukte nachweisen [28]. In den ersten $24 \mathrm{~h}$ wird soviel Addukt gebildet, wie bei einer $20 \%$ Freisetzung von Benzidin aus dem Farbstoff zu erwarten wäre.

Zahlreiche Azo-Pigmente enthalten 3,3'Dichlorbenzidin als Kupplungskomponente, und es kann mit Hilfe des HämoglobinAdduktes geprüft werden, ob die Azo-Gruppen in diesen praktisch unlöslichen Pigmenten in vivo reduktiv gespalten werden können. Da wegen der Atembarkeit von Stäuben auch die Lunge als Eintrittspfad zu be- rücksichtigen ist, wurde Pigment Yellow 17 bei der Ratte intratracheal instilliert und nach $7 \mathrm{~d}$ das Blut auf Hämoglobin-Addukte von 3,3'-Dichlorbenzidin untersucht. Bei einer Nachweisempfindlichkeit von $c a$. $0.004 \%$ der Dosis konnte kein Addukt nachgewiesen werden [29].

Auch nach einmaliger oraler Applikation der radioaktiv markierten Pigmente Yellow 13 und 174 wurden nach $48 \mathrm{~h}$ weder Hämoglobin-Addukte im Blut noch Metaboliten im Harn gefunden [30]. Diese Befunde sprechen dafür, dass die kanzerogene Komponente im Gegensatz zu besser löslichen Azo-Farbmitteln aus diesen Azo-Pigmenten unter den angewandten Bedingungen - wenn überhaupt - nur in ganz geringen Mengen freigesetzt wird. Um die Bioverfügbarkeit experimentell noch zwingender auszuschliessen ist es erforderlich, die Pigmente chronisch, z.B. mit dem Futter über längere Zeit zuzuführen.

\section{Risikobeurteilung}

In Kenntnis dieser Ergebnisse hat der Ausschuss für Gefahrstoffe (AGS) in der Bundesrepublik Deutschland die Azo-Pigmente bereits von der Regelung für Azofarbmittel mit kanzerogenen Komponenten ausgenommen. Es wäre aber dennoch dringend zu empfehlen, ausser den beruflich gegenüber hydrophilen und lipophilen Azofarbstoffen Exponierten auch Pigment-Exponierte in den Kreis derjenigen einzubeziehen, bei denen die Exposition über Hämoglobin-Addukte im Blut kontolliert wird. Damit liesse sich eine Belastung des Menschen beim Umgang mit Azo-Pigmenten am überzeugendsten ausschliessen.

Über die Expositionskontrolle bei bekannten Expositionen hinaus, gestattet es die Analyse der Hämoglobin-Addukte, die aus verschiedenen - auch unbekannten Quellen stammende und über verschiedene Pfade aufgenommene Menge eines Amins zu erfassen. Dadurch kann zunächst der Beitrag eines bestimmten Amins zur Gesamtbelastung durch gentoxische Amine abgeschätzt werden. In einem weiteren Schritt gilt es dann mit Hilfe dieses und anderer Biomonitoring-Verfahren den Beitrag von Arylaminen und Nitroaromaten mit der Gesamtbelastung durch gentoxische Stoffe in Beziehung zu setzen. Erst auf dieser Grundlage wird es gelingen, das kanzerogene und erbgutschädigende Potential abzuschätzen und vernünftig einzuordnen [31].

Die Erfahrungen mit Arylaminen zeigen, dass auch akut toxische Effekte einen Beitrag zur Kanzerogenität leisten können. Beides, die Abschätzung des Beitrags einer Substanz zum Gesamt-DNA-Schaden und die Abschätzung von Expositionen, die zu einer relevanten Beeinträchtigung physiologischer Gleichgewichte führen, werden Voraussetzung dafür sein, gesundheitlich unbedenkliche oder gesellschaftlich akzeptierbare Grenzwerte festzulegen. 
[1] H. Letheby, Proc. R. Soc.London. [Ser.] A 1863 12, 550 .

[2] W. Rayner, Br.Med.J.1886, I,294; P.Borinski, Disch. Med. Wochensehr. 1921, 47, 1526.

[3] K. Klingenberg, Dissertation, Rostock, 1891.

[4] J. Trefouel, F. Nitti, D. Bovet, C.R. Seances Soc. Biol. 1935, 120,765.

[5] T. Hashimoto, Gann 1935, 29, 306.

[6] W.J.P. Neish, Natuwissenschaften 1959,46,535.

[7] G.C. Mueller, J.A. Miller, J. Biol. Chem. 1948 , 176, 535 .

[8] J.C. Radomski, T.J. Mellinger, J. Pharmacol. Exp. Ther. 1962, 136, 259

[9] M. Kiese, Naunyn-Schmiedeberg's Arch. Exp Pathol. Pharmakol. 1959, 235, 354.

[10] J.W. Cramer, J.A. Miller, E.C. Miller, J. Biol Chem. 1960, 235, 885

[11] I. Rehn, Arch. Klin. Chir. 1895, 50, 588

[12] B. Fischer, Münch. Med. Wschr. 1906, 2041; M B. Schmidt, Virchow's Arch. Path. Anat. 1924 253,432 .

[13] T. Yoshida, Gann 1935, $28,454$.
[14] H. Druckrey, K. Küpfmüller, Z. Naturforsch., B 1948, 3, 254

[15] R. Oppenheimer, Z. Urol. Chir, 1927, 21, 336.

[16] S. Spitz, W.H. Maguigan, K. Dobriner, Cancer $1950,3,789$.

[17] B. Walker, A. Gerber, Natl. Cancer Inst. Monograph 1981, 58, 11 .

[18] R. Anliker, D. Steinle, $J$. Soc. Dyers Colour 1988, $104,377$.

[19] E. Rinde, W. Troll, J.Natl.CancerInst. 1975,55, 181.

[20] J.F. Robens, G.S. Dill, J.M. Ward, R.A. Griesemer, J.F. Douglas, Toxicol. Appl. Pharmacol. $1980,54,431$.

[21] W.A. Genin, Vopro Onkol. 1977, 23, 50; M. Boeniger, DHHS (NIOSH) Publ. No. 80-109, 1980.

[22] ETAD Bericht T 2028-B, 1989.

[23] Mitteilung XXVI der Senatskommission zur Prüfung gesundheitsschädlicher Arbeitsstoffe, VCH Verlagsgesellschaft, Weinheim, 1990.

[24] B. Dölle, W. Töpner, H.-G. Neumann, Xe- nobiotica 1980, 10,527; P. Eyer, E. Lierheimer, ibid. 1980, 10, 517; H.G. Neumann, Arch. Toxicol. 1984, 56, 1 .

[25] H.M. Bolt, H.-G. Neumann, J. Lewalter, Arbeitsmed. Sozialmed. Prärentivmed. 1985, 20. 197.

[26] J. Lewalter, U. Korallus, Im. Arch. Occup Environm. Health 1985, 56, 179.

[27] G. Birner, H.-G. Neumann, Arch. Toricol. 1988, $62,110$.

[28] G. Birner, W. Albrecht, H.-G. Neumann, Arch. Toxicol. 1990, 64, 97

[29] I. Zwirner-Baier, H.-G. Neumann, unveröffentlicht.

[30] P. Sagelsdorf, R. Joppich-Kuhn, M. Joppich, $J$. Cancer Res. Clin. Oncol. 1990, Suppl. Vol. $/ 16$, 79.

[31] H.-G. Neumann, IARC Scicntific Pıbl. 1988, No. 89,157 ; H.-G.Neumann, Chenic in wnserer Zeit $1991,25,102$.
Chimia 45 (1991) 303

(C) Schweiz. Chemiker-Verband; ISSN 0009-4293

\section{Die objektive und subjektive Bedeutung der Farbe}

\section{Max Lüscher*}

\section{Die objektive Bedeutung der Farben}

Farben wären nicht interessant, wenn wir sie nicht wahrnehmen und erleben könnten.

Jede Farbe, die wir wahmehmen bewirkt eine bestimmte Sinnesempfindung. Die Sinnesempfindung von Rot wird anders erlebt als die von Grün oder Blau. Es besteht kein Meinungsunterschied, welche Farbe rot und welche blau ist, oder welche gelbrot und welche blaurot erscheint. Das bedeutet, dass jeder der eine bestimmte Farbe wahrnimmt, sie genau gleich empfindet wie jeder andere. Jede Farbe hat deshalb ihre allgemeingültige objektive Bedeutung.

Die Farbpsychologie beschreibt und definiert die objektive psychologische Bedeutung der einzelnen Farben.

\section{Die subjektive Bedeutung der Farben}

Um Missverständnissen sogleich zu begegnen sei erwähnt, dass die Psychologie zwei radikal verschiedene Definitionen zu geben hat:

1. die objektive Bedeutung der Farbempfindung und

2. die subjektive persönliche Einstellung des Einzelnen zu dieser Farbe. Ob der Einzelne diese Farbe sympathisch, gleichgültig oder unsympathisch findet, das ist seine persönliche Bewertung. Die persönliche Ein-

\footnotetext{
* Korrespondenz: Prof. Dr. M. Lüscher
} Kreuzbuchrain 14, CH-6006 Luzern stellung zeigt, welches Gefühl jemand bei dieser Farbe hat. Ein Beispiel soll das deutlich machen: Die objektive Bedeutung der Sinnesempfindung Rot ist für jedermann in jeder Kultur: Erregung. Ob jemand die Erregung von Rot als angenehmen Reiz lustvoll erlebt oder ob ein anderer dasselbe Rot als aufreizend und unangenehm aufregend findet, das ist individuell verschieden.

Noch ein zweites Beispiel: Dunkelblau hat die objektive Bedeutung: Ruhe. Wer Dunkelblau mag, liebt Ruhe. Wer es nicht mag, erträgt die Ruhe nicht. Er empfindet sie als langweilige Leere. Darum ist er ruhelos.

\section{Farben sind allgemeingültige Signale}

Wer eine Farbe wahrnimmt, erlebt ihre objektive Bedeutung. Jede Farbe ist deshalb ein genau bestimmbares emotionales Signal. Es wird unbewusst erlebt. Farbsignale sind eine emotionale Sprache, die unbewusst verstanden wird. Sie ist im Gegensatz zur verbalen Sprache erstens allgemeingültig und zweitens eindeutig. Abstrakte Worte hingegen wie Liebe, Gerechtigkeit, Anstand sind vieldeutig.

\section{Die Signalsprache der Farben}

Die emotionale Sprache der Farben ist nicht nur allgemeingültig sondern auch zwingend. Wer eine Farbe wahrnimmt, erlebt gleichzeitig ihre emotionale Wirkung.

Das macht sich die Werbung zu Nutzen.
Zigarettenmarken, Genuss und Waschmittel können wegen der Farbe der Packung zu Bestsellern werden. Wenn jemand eine bestimmte Benzinmarke bevorzugt, kann es nur wegen des farbigen Markendesigns sein, denn er kann die Qualität des Benzins nicht beurteilen.

Die Farbe in der medizinischen Diagnostik

Derpsychologische «Klinische Farbtest» wird zur Messung des psychisch-somatischen Verhaltens in verschiedenen Persönlichkeitsbereichen verwendet. Bestimmte Arten von chronischem Stress, zum Beispiel Ängstlichkeit, Ruhelosigkeit, Ärger belasten bestimmte Funktionssysteme und führen schliesslich zu bestimmten Erkrankungen.

Mit statistischer Signifikanz wurden zum Beispiel die Farbwahlen für Herzinfarktdisposition, für Bluthochdruck, Magengeschwür, Fettsucht, Alkoholismus, Suizid usw. festgestellt.

Verbreitung hat der «Klinische Farbtest» auch unter Zahnärzten gefunden. Dazu zwei Beispiele: Das bläulich Test-Grün (2) wird im Durchschnitt von $4 \%$ abgelehnt. Nächtliche Knirscher lehnen es hingegen zu 55,88\% ab. Schwarz wird im Durchschnitt von nur $2 \%$ bevorzugt. Jugendliche mit Kieferanomalien und schrägstehenden Zähnen hingegen bevorzugen es zu $36 \%$.

Die einzelnen Symptome sind Ausdruck von gestörten und erkrankten Funktionssystemen. (In der Akupunktur werden sie als Meridiane bezeichnet.) Aus den Farbwahlen können solche Störungen und psychischsomatische Fehlhaltungen erkannt werden. Darum dient der «Klinische Farbtest» der medizinischen Diagnostik und kann zur kostensparenden Früherkennung zum Beispiel der Disposition zum Herzinfarkt verwendet werden.

Die Testfarben haben es erstmals möglich gemacht, das psychisch-somatische Befinden des Patienten zu messen. 